\title{
Don Quijote y la novela histórica: Consideraciones sobre la influencia de la nove- la cervantina en Waverley, or 'Tis Sixty Years Since (I8I4), de Sir Walter Scott
}

Alfredo Moro Martín

$\mathrm{E}$ del pasado:

Salí de mi patria, empeñé mi hacienda, dejé mi regalo y entrégueme en los brazos de la fortuna, que me llevasen donde más fuese servida. Quise resucitar la ya muerta andante caballeria, y ha muchos días que tropezando aquí, cayéndome allí, despeñándome acá y levantándome acullá, he cumplido gran parte de mi deseo, socorriendo viudas, amparando doncellas y favoreciendo casadas, huérfanos y pupilos, propio y natural oficio de caballeros andantes; y así, por mis valerosas, muchas, y cristianas hazañas, he merecido andar ya en estampa en casi todas o las más naciones del mundo: treinta mil volúmenes se han impreso de mi historia, y lleva camino de imprimirse treintamil veces de millares, si el cielo no lo remedia. (2.16:662-663; énfasis añadido)

La voluntad de don Quijote de "resucitar la ya muerta andante caballería" subraya el contraste entre sus nostálgicas aspiraciones y un tiempo bien distinto, en el que los caballeros andantes y sus usos conservan una vigencia muy limitada, o incluso pertenecen únicamente al ámbito 
de lo literario, de la memoria libresca de los lectores y algunos personajes de la novela. ${ }^{1}$ El carácter anacrónico de las aspiraciones quijotescas ha sido puesto de relieve en numerosas ocasiones tanto por los distintos lectores de Don Quijote como por la crítica cervantina. En el siglo XIX, John Gibson Lockhart, autor de la introducción a la reedición de la traducción del Quijote llevada a cabo por Peter Motteux a comienzos del siglo XVIII (I700-I703), ya resaltaba cómo "the practice of knight-errantry, if ever there was such a thing, had [...] been out of date long before the age in which don Quixote appeared' (Introduction I822: lvi; énfasis añadido), mientras que Georg Wilhelm Friedrich Hegel hablaba en sus Vorlesungen über die Ästhetik (I8I8-I8I9) de una "Auflösung des Rittertums in sich selbst" [una disolución de la caballería en sí misma] tanto en Ludovico Ariosto como en Miguel de Cervantes (Werke I4: 2I7), apuntando cómo el autor español representa en su Quijote la caballería y su concepción individualista de la justicia como cosa anacrónica (Werke 13: 257). ${ }^{2}$ Por lo que respecta a la crítica cervantina,

I En opinión del crítico chileno Félix Martínez-Bonati, el mundo caballeresco no se encuentra presente en la novela de Cervantes y posee un carácter puramente referencial: "Acontecimientos característicos del mundo caballeril no tienen lugar en el mundo de don Quijote. Meramente se los evoca, en especial por medio de las erradas interpretaciones que hace el protagonista de sus circunstancias, y por las bien o mal intencionadas mentiras y simulaciones de amigos y extraños. Lo caballeril es sólo parte de la imaginación de los personajes y de la memoria de los lectores. [...] Dentro de la ficción que es el Quijote, lo caballeril es sólo una fantasía, de constante presencia, pero nada más que imaginada y libresca, una nube ideal sobre el mundo "real"; es una región literaria no encarnada en el estrato mundanal básico de la obra" (I92; énfasis añadido).

2 En sus Vorlesungen über die Ästhetik, Hegel traza un interesante paralelismo entre Don Quijote y el Götz von Berlichingen mit der eisernen Hand (I773) de Goethe. En lo que se refiere a la concepción de la justicia, Hegel señala como Goethe y Cervantes comparten el retrato de una concepción caballeresca y heroica de la justicia situada en un tiempo moderno en el que predomina una idea más prosaica y moderna de la misma. El contraste entre ambas reflejaría el carácter medieval, y consecuentemente anacrónico, de los protagonistas de ambas obras, siendo este anacronismo dotado de una mayor 
Ramón Menéndez Pidal, ya en el siglo XX, en su discurso de clausura de la asamblea cervantina de 1948, titulado "Cervantes y el ideal caballeresco" destacó cómo "el noble caballero de la Mancha quería traer de nuevo a la vida un pasado desaparecido" (26; énfasis añadido), mientras que un ilustre cervantista e historiador de la caballería como Martín de Riquer se ha referido a don Quijote como un "anacronismo viviente" o "un paradigma de lo inactual" ("Introducción” lxxiv). ${ }^{3}$ Más recientemente, Heinz-Peter Endress, en su estudio Los ideales de don Quijote en el cambio de valores de la Edad Media hasta el Barroco (2000) ha puesto de relieve la importancia de la "dislocación temporal" de la locura quijotesca, que propicia el empeño de Alonso Quijano por "resucitar en la actualidad, es decir, hacia I600, una especie legendaria de caballeros andantes, extinguida hace mucho tiempo [...] y de hacer así de nuevo justicia a una época irrecuperablemente desaparecida" (I8; én-

comicidad en el caso de Cervantes (ver Hegel 257). Como se podrá apreciar a lo largo de este artículo, las ideas de Hegel en torno a Cervantes, Goethe y el retrato que ambos realizan de una época de transición entre dos tiempos distintos, se corresponden de manera bastante precisa con las de Scott en torno al Quijote, y este dato no resulta del todo sorprendente ya que la primera obra literaria firmada por el autor escocés fue precisamente una traducción al inglés del Götz von Berlichingen de Goethe, que aparecería en Londres en 1799 con el título de Götz von Berlichingen with the Iron Hand.

3 Ya en 1973, en su artículo "Cervantes y la caballeresca," Martín de Riquer subrayaba el carácter anacrónico del hidalgo manchego: "Ya es sabido que la libresca locura de don Quijote se manifiesta, entre otras cosas, por la nostalgia de un tiempo pasado en que existía la caballería andante "novelescamente», ya que, como hemos visto, sólo un siglo antes España contaba con numerosos caballeros andantes de veras. El hidalgo manchego es, esencialmente, un arcaísmo viviente que deambulará por los caminos de Espańa a principios del siglo XVII. Uno de los muchos aspectos que más fácilmente pierde el lector moderno es el singular atuendo militar de don Quijote. [...] Este aspecto exterior de don Quijote produce o bien la risa o bien la estupefacción de cuantos encuentra por el camino, impresión similar a la que hoy nos produciría ver un batallón de paracaidistas mandado por un comandante que vistiera el uniforme del general Prim en la guerra de Marruecos" (“Cervantes" 289; énfasis añadido). 
fasis añadido). En opinión del crítico germano, esta dislocación temporal del caballero manchego permite a Cervantes "simbolizar de manera pragmática el cambio que opera en los valores desde la Edad Media al Barroco," reflejando, por lo tanto, un claro contraste entre épocas históricas bien distintas (I3). Por su parte, Pedro Cátedra, en su libro de 2007, El sueño caballeresco, ha señalado cómo don Quijote "es como un ente cultural sacado de su contexto y abandonado a su suerte" en un nuevo tiempo en el que los códigos caballerescos poseen una validez únicamente referencial (8). ${ }^{4}$

Quizás el análisis más concienzudo y detallado de este contraste entre lo que Heinz-Peter Endress llama la utopía restaurativa del pasado caballeresco que don Quijote trata de materializar y los tiempos modernos ha sido ofrecido por el profesor Francisco Vivar en su magnífico estudio Don Quijote frente a los caballeros de tiempos modernos, publicado en Salamanca en 2009 (Endress 53).5 Para Vivar, don Quijote no es

4 Maurice Keen, en su magnífico ensayo La caballería (1986), subraya cómo a lo largo de todo el siglo XVI se van a producir importantes cambios en la orden de la caballería: "Más importante que todo esto eran los cambios que en el siglo XVI estaban teniendo lugar en un nivel más profundo y que estaban alterando las estructuras sociales y políticas en las que la caballería había florecido en el pasado. Lo que vemos al final de la Edad Media es, en consecuencia, no tanto la decadencia de la caballería, sino el cambio de su aspecto, y ello se advierte en la apreciación de las grandezas de la caballería, así como de sus locuras, que Cervantes se complace en presentar en el Quijote. Los ánimos que en la época medieval habían dado vida e ímpetu a la caballería se hallaban todavía en vigor, pero los aspectos en los que encontraban su modo de expresión estaban cambiando y el antiguo nombre perdiendo su propiedad" (3I4). De las palabras de Keen se desprende cómo, a principios del siglo XVII, la caballería, al menos tal y como la entiende don Quijote, contaba únicamente con un valor ético/moral o referencial, como acertadamente apunta Cátedra. A comienzos del XVII los caballeros andantes formaban parte del universo de la literatura, y no tanto del mundo real.

5 Por utopia restaurativa, Endress se refiere "al movimiento de avance y retroceso que ensarta lo pretérito con lo futuro y a la diferencia existente frente a los proyectos habituales emplazados en otro lugar o encauzados solamente hacia el futuro" (53). 
hijo, sino más bien "hijastro" de su tiempo, ya que "la existencia de don Quijote no se corresponde con la historia, su mundo interior ya no tiene mucho que ver con el exterior" (I3; énfasis añadido). En opinión del profesor español, la segunda parte de la obra cervantina ofrece un claro contraste entre el anacrónico mundo de la locura quijotesca y la situación de España a comienzos del siglo XVII, siendo esta antítesis subrayada por la contraposición dialógica entre don Quijote y los caballeros que sí viven dentro de la historia: don Diego de Miranda, Camacho el Rico, los Duques, Roque Guinart y Antonio Moreno. Vivar considera que estos encuentros propician un proceso de autoconocimiento por parte de don Quijote, que culminaría en la renuncia a los libros de caballería y a su utopía restauradora, reconciliando a Alonso Quijano con su realidad histórica justo antes de morir (I44). ${ }^{6}$

Si se tiene en cuenta que, en su ensayo The Historical Novel, Georg Lukács consideraba este contraste dialógico entre fuerzas sociales e históricas distintas como uno de los puntales estructurales de la novela histórica tal y como la entiende Sir Walter Scott, padre indiscutible del género, resulta sorprendente la poca atención crítica que tanto el cervantismo como los estudiosos de la novela histórica han dedicado al

6 En opinión de Edwin Williamson, la locura de don Quijote muestra dos dimensiones bien distintas. Por una parte estaría su "creencia en la absoluta veracidad de los libros caballerescos" y por otra "la convicción de que él mismo está destinado a restaurar la caballería" (39; énfasis añadido). Para el cervantista escocés, don Quijote nunca abandona "la creencia fundamental de que los libros no pueden mentir" (39), pero a lo largo de la segunda parte, y fundamentalmente a partir la aparición de Dulcinea como labradora en el décimo capítulo de la segunda parte, don Quijote comienza a tener dudas acerca de su destino caballeresco, disminuyendo este segundo grado de locura y hundiéndose en un proceso de agnición, es decir, en un paulatino "cambio desde la ignorancia al conocimiento" (44). En mi opinión, esta transformación de don Quijote a través de la desilusión es claramente reconocible a lo largo de la segunda parte, pero creo que este proceso se ve impulsado también por el contraste con los caballeros de tiempos modernos y el consecuente reconocimiento de la inadecuación histórica de la vocación caballeresca, tal y como Francisco Vivar propone en su estudio. 
papel de Don Quijote en la gestación de este género novelístico.7 Hasta la fecha, no tengo constancia de estudios procedentes del ámbito del cervantismo que hayan tratado la influencia de Don Quijote en la novela histórica, aunque algunos investigadores especializados en este género como Jerome de Groot o Brian Hamnett sí que se han referido puntualmente al papel jugado por Cervantes en la prehistoria de la novela histórica. Para de Groot, "Don Quixote dramatises the ways in which fictions of the past might infect the present and lead to romantised madness, how accounts of history can have an effect on the contemporary mind" (13), algo que situaría al Quijote como una clara influencia en el género que nos ocupa (I4); mientras que Hamnett ha destacado cómo don Quijote se encuentra claramente "unreconciled to modern times," reconociendo cómo la novela cervantina, además de ser un ensayo sobre los peligros de la imaginación, también supone un importante referente para la novela histórica decimonónica (23).

7 Para Lukács, las novelas históricas de Scott siguen un principio estructural básico. El protagonista, habitualmente un anti-heroico burgués inglés más o menos mediocre, se ve envuelto en la confrontación entre dos movimientos históricos y sociales antagónicos. Su posición dubitativa e intermedia le permite observar esta confrontación y el progreso histórico que resulta de la derrota de las fuerzas históricas anacrónicas y pertenecientes al pasado, un proceso que Lúkacs denomina declassing (The Historical Novel 34): "Scott's heroes, as central figures of the novel, have an entirely opposite function. It is their task to bring the extremes whose struggle fills the novel, whose clash expresses artistically a great crisis in society, into contact with one another. Through the plot, at whose centre stands this hero, a neutral ground is sought and found upon which the extreme, opposing social forces can be brought into a human relationship with one another" (36). Por su parte, Román Álvarez, en su estudio de 1983 sobre la novela histórica inglesa, señalaba, siguiendo a Lukács, cómo Scott se sirve del pasado para "situar frente a frente dos épocas, dos culturas o dos fuerzas históricas" (69), retratando "los momentos cruciales de la Historia, es decir, las épocas de profundo cambio, o los momentos en que culturas distintas entran en fricción” (74). La novela histórica, por lo tanto, cuenta, como Don Quijote, con un principio estructural fundamentalmente contrastivo y dialógico. 
La carestía de estudios que vinculen Don Quijote con el florecimiento de la novela histórica a finales del siglo XVIII resulta aún más sangrante si consideramos la reconocida admiración por Cervantes de Sir Walter Scott, cuya primera novela, Waverley, or 'Tis Sixty Years Since (I8I4) es habitualmente considerada como el punto de partida del género. Las referencias del autor escocés a Cervantes y al Quijote son habituales no sólo en su producción novelística y ensayística, en la que podemos encontrar numerosas citas del Quijote, de las Novelas Ejemplares e incluso del Persiles, sino también en su correspondencia y en sus diarios, en los que habitualmente alude al autor español y su obra (Snel-Wolfe 30I-II). En este sentido, resulta especialmente significativo el papel que Cervantes y Don Quijote juegan en los Essays on Chivalry, Romance, and the Drama (I815-1824), en los que el novelista escocés se ocupa del nacimiento, desarrollo y declive de la caballería y su manifestación narrativa, la ficción caballeresca. Para Scott, resulta evidente que don Quijote de la Mancha es un hombre fuera de su tiempo. Así, el novelista escocés destaca cómo "the Knight of La Mancha was, perhaps, two centuries too late in exercising his office of redresser of wrongs," aunque matiza cómo la España del XVII todavía conservaba el halo romántico de los tiempos del pasado, ya que, "at no great distance from the date of the inimitable romance of Cervantes, real circumstances occurred, of a nature as romantic as the achievements which Don Quixote aspired to execute" (Essays 85-86). En otras palabras, don Quijote es para Walter Scott un sujeto fuera de su contexto histórico, pero dentro de un horizonte referencial que todavía le permite soñar con resucitar la andante caballería. ${ }^{8}$ En los Essays on Chivalry, Romance, and the Drama, Scott parece por lo tanto entender

8 En este sentido, McDonald Jr ha señalado cómo Scott plausibiliza el comportamiento de don Quijote como un vestigio de un tiempo feudal que poco a poco se desvanece en el presente: "Thus, while acknowledging the over-developed imagination of the hero, Scott nevertheless affirmed that the Knight's character and actions had some basis in actuality, as did the romances; and elsewhere in the same essay he declared that the defiance which Quixote hurled against the Merchants of Toledo had its prototype in the opposition of the Knights to the encroachment of the King's taxes" (4I; énfasis añadido). 
Don Quijote como una novela que retrata la transición entre dos tiempos históricos distintos: el mundo feudal que la utopía restaurativa de don Quijote trata de resucitar y el mundo moderno al que la España del XVII se encamina, en el que los usos caballerescos conservan una vigencia únicamente referencial, erigiéndose como un recuerdo de una época ya superada. De hecho, para Walter Scott, uno de los principales factores en la desaparición definitiva de las costumbres caballerescas en España será la propia publicación de Don Quijote:

The romances of Chivalry, did not, however, sink into disrepute under the stern rebuke of religious puritans or severe moralists, but became gradually neglected as the customs of chivalry itself fell into disregard; when of course the books which breathed its spirit, and were written under its influence, ceased to produce any impression on the public mind, superseded by better models of composition, and overwhelmed with the ridicule of Cervantes, sunk by degrees into utter contempt and oblivion. (I88-I89) ${ }^{9}$

Esta opinión sobre la pervivencia y paulatina desaparición de los usos caballerescos en la España de Don Quijote se ve ratificada por otro ensayo posterior de Scott, sus Lives of the Novelists (I82I-I824), en el que el autor escocés pasa revista a la biografía y obra de autores como Henry Fielding, Tobias Smollett o Alain-René Lesage, todos ellos de reconocida raigambre cervantina. Al analizar el uso de figuras quijo-

9 En este sentido, cabe destacar cómo la opinión de Scott coincide con la de otra figura del Romanticismo británico, Lord Byron. En el canto XIII de su Don Juan, el poeta inglés sugiere que la publicación de Don Quijote acabó con el espíritu caballeresco en España, y por ende, con su gloria imperial: "Cervantes smiled Spain's Chivalry away; / A single laugh demolished the right arm / Of his own country; — seldom since that day / Has Spain had heroes. While Romance could charm, / The world gave ground before her bright array; / And therefore have his volumes done such harm, / That all their glory, as a composition, / Was dearly purchased by his land's perdition" (768). 
tescas por parte de Fielding y Smollett, Scott significativamente critica a su compatriota por el uso de un moderno Quijote en The Life and Adventures of Sir Launcelot Greaves (1760). Para Scott, la introducción de un segundo Quijote en la Inglaterra del siglo XVIII resulta poco realista, ya que en la Inglaterra dieciochesca el remanente caballeresco que poco a poco se extinguía en la Espańa del siglo XVII se encontraba ya totalmente extinto:

The leading imperfection is the great extravagance of the story, as applicable to England, and to the period when it is supposed to have happened. In Spain, ere the ideas of chivalry were extinct amongst that nation of romantic hidalgos, the turn of Don Quixote's frenzy seems not altogether extravagant, and the armour which he assumed was still the ordinary garb of battle. But in England, and in modern times, that a young amiable, and otherwise sensible man, acquainted also with the romance of Cervantes, should have adopted a similar whim, gives good foundation for the obvious remark of Ferret: "What! You set up for a modern Don Quixote! The scheme is too stale and extravagant; what was an humorous and well-timed satire in Spain near two hundred years ago will make but a sorry jest, when really acted from affectation, at this time of day in England." (52-53)

Scott vincula de este modo la posibilidad de recrear a un moderno don Quijote al lugar y a las circunstancias históricas, lo que nos revela su entendimiento del quijotismo como un fenómeno que debe de ser enmarcado en un tiempo histórico concreto, en unas coordenadas espacio-temporales muy determinadas. En este artículo, pretendo analizar la continuidad de estos presupuestos teóricos en la primera novela del autor escocés, Waverley, or 'Tis Sixty Years Since, cuya filiación cervantina es indiscutible, y en la que se puede encontrar un uso del quijotismo muy vinculado al contraste dialógico entre un tiempo heroico y feudal irremisiblemente caduco, y unos tiempos modernos caracterizados por 
su carácter burgués y quizás poco romántico. ${ }^{10}$ Inglaterra, por su espectacular desarrollo socioeconómico durante el siglo XVIII, no ofrecía las características idóneas para retratar este contraste. Algo muy distinto ocurrirá con Escocia, en la que los intentos jacobitas por resucitar una visión del mundo que poco a poco comenzaba a apagarse ofrecerán a Scott la oportunidad ideal para representar artísticamente esta confrontación entre dos visiones de la realidad y de la historia totalmente antagónicas, dando forma a lo que la crítica posterior considerará la primera novela histórica. Como trataré de demostrar a continuación, esta confrontación dialógica entre el pasado y el presente, característica fundamental de la novela histórica tal y como la entiende Lukács, cuenta en Scott con un claro regusto cervantino, y es de hecho el resultado directo de la elección de un protagonista de trazos quijotescos por parte del autor escocés. La fascinación quijotesca por el pasado y el

Io El reconocimiento de la filiación cervantina de Waverley cuenta con un largo recorrido crítico, aunque no son demasiados los estudios que han profundizado en las implicaciones de esta influencia respecto a la cuestión del origen de la novela histórica como género. Alexander Welsh (Reflexions), Theodor Wolpers, Wolfgang G. Müller, y Heinz-Joseph Müllenbrook han abordado desde diferentes perspectivas el papel que Don Quijote juega en la primera novela de Scott. Más recientemente, Pedro Javier Pardo ha analizado la influencia de Don Quijote en Waverley desde el prisma de la conexión que surge a finales del siglo XVIII y principios del XIX entre literatura de viajes y literatura cervantina; mientras que Moro ha reivindicado el papel del autor escocés dentro de la recepción de Cervantes en las letras británicas ("Cervantismos olvidados"). Las primeras décadas del siglo XXI han visto un aumento de los estudios por parte de la crítica cervantina respecto a la novelística de Scott, y las contribuciones de Robert ter Horst, Michael Gerli y Howard Mancing reflejan este creciente interés. Ter Horst ha analizado las afinidades entre la obra de Cervantes y la del autor escocés, así como su similar relación inclusiva respecto al romance. Por su parte, Gerli ha estudiado la utilización por parte de Scott en relación a sus propios planteamientos sobre la legitimidad del género de la novela a principios del siglo XIX; mientras que Mancing, limitado al contexto de un capítulo sobre la recepción de Cervantes en Gran Bretańa durante los siglos XIX y XX, destaca la fascinación del autor escocés por Cervantes y ciertos paralelismos entre la obra de ambos autores. 
retrato de su inexorable decadencia van a estar profundamente ligados en Waverley, reflejando cómo la sombra de Cervantes se extiende al amanecer de la novela histórica y a los inicios de Sir Walter Scott como novelista.

\section{WAVERLEY Y EL CONTRASTE CERVANTINO ENTRE PASADO Y PRESENTE}

En Waverley, or 'Tis Sixty Years Since, Walter Scott se ocupa del conflicto armado que tuvo lugar en 1745 entre los partidarios de la dinastía Estuardo, que había dejado el trono de Gran Bretańa en I7I4 como consecuencia de la Revolución Gloriosa, y la dinastía reinante, los Hanover, que ocuparían el trono británico en adelante. En Escocia, donde los Estuardo habían permanecido en el poder desde el siglo XIV hasta el final del reinado de Jacobo II (I685-I688) —último monarca Estuardo de Inglaterra y Escocia-, la rebelión jacobita resultó especialmente virulenta, ya que los Estuardo contaban con un número considerable simpatizantes deseosos de recuperar un régimen absolutista de tintes feudales, así como las viejas libertades escocesas, parcialmente perdidas con la Act of Union de 1707. Scott sitúa en este contexto histórico al protagonista de su novela, Edward Waverley, ya que las particulares circunstancias históricas de Escocia e Inglaterra a mediados del siglo XVIII van a resultar determinantes tanto en la peculiar educación que el protagonista recibe y de la que Scott se ocupa en detalle, como en el ulterior desarrollo de la novela. Concretamente, en el segundo capítulo de la obra se narra cómo el padre de Edward, Richard Waverley, decide asumir una serie de responsabilidades parlamentarias al servicio de la monarquía hanoveriana, circunstancia que le fuerza a permanecer en Londres durante una gran parte del año, obligándole a delegar la educación de su hijo en su hermano, Sir Everard Waverley, más en línea con la tradicional simpatía de la familia por la monarquía Estuardo y la causa jacobita. Esta desatención paterna acarrea una serie de deficiencias en la educación de Edward, especialmente una vez que su tío decide encargar el cuidado educativo de su sobrino a un capellán de claras convicciones jacobitas, pero ya entrado en edad y poco dispuesto 
a ejercer cualquier tipo de autoridad sobre su pupilo, hecho que conlleva que Edward aprenda "as he pleased, what he pleased, and when he pleased" (Waverley I.3: II). Esta falta de disciplina propicia que el protagonista dirija su interés hacia toda una serie de lecturas, que Scott se encarga de enumerar minuciosamente, caracterizadas todas ellas por su naturaleza fundamentalmente romántica. Así, Edward, en la biblioteca gótica de Waverley Hall, navegará "through the sea of books like a vessel without a pilot or a rudder" (I.3: I3), empleando sus horas en la lectura de los dramaturgos isabelinos, de las crónicas caballerescas francesas de Jean Froissart (I337-I4O4), Pierre de Brantôme (I54O-I6I4) o François de la Noue (I53I-I59I); o incluso disfrutando de la literatura española, que en palabras del narrador "contributed to his stock of chivalrous and romantic lore" (I.3: I4). Estas lecturas despiertan un carácter imaginativo y solitario, poco amigo de la sociedad circundante, mucho más dispuesto a pasar horas junto a sus tíos, y siempre atento a su relato de las viejas gestas familiares. Edward disfruta escuchando a Sir Everard relatar las hazañas de Willibert de Waverley en las Cruzadas y las románticas circunstancias de su vuelta a Inglaterra; así como de la narración que su tía hace de la resistencia heroica de Lady Alice Waverley durante la Guerra Civil inglesa, en la que permaneció del lado de los Estuardo (I.4: 16). Esta afición a la lectura y a los relatos familiares del protagonista pronto se transforma en un gusto por reconstruir imaginativamente en los dominios familiares las escenas caballerescas del pasado narradas por Sir Everard y Mrs Rachael Waverley, hábito que conlleva que la vivencia de este "ideal world" sea cada vez más delectable para el joven inglés (I.4: I7). La educación romancesca del protagonista, propiciada por los modelos literarios que Scott se encarga de describir en detalle, se ve por lo tanto teñida de las simpatías jacobitas de sus tíos, siempre celosos de asociar el glorioso pasado de los Waverley con la lealtad a la dinastía que ellos consideran legitimada para reinar en Gran Bretaña. Se crea de esta manera un vínculo claro entre literatura y política, entre un gusto literario concreto y una visión muy determinada sobre el problema dinástico escocés, y esta relación va a tener, como se podrá observar a continuación, una importancia fundamental en la novela de Scott. 
Los paralelos de la educación del joven Waverley con la figura quijotesca no se escapan al narrador de la novela, que, si bien reconoce el claro pedigrí cervantino de su protagonista, matiza el parecido entre ambas figuras que los capítulos tercero y cuarto despiertan en el lector:

From the minuteness with which I have traced Waverley's pursuits, and the bias which they unavoidably communicated to his imagination, the reader may perhaps anticipate, in the following tale, an imitation of the romance of Cervantes. But he will do my prudence injustice in the supposition. My intention is not to follow the steps of that inimitable author, in describing such total perversion of intellect as misconstrues the objects actually presented to the senses, but that more common aberration from sound judgement, which apprehends occurrences indeed in their reality, but communicates to them a tincture of its own romantic tone and colouring. (I.5: I8; énfasis añadido)

El narrador, por lo tanto, reconoce los paralelos con la figura quijotesca, pero se distancia al mismo tiempo de la novela cervantina, destacando cómo no estamos ante un demente, sino más bien ante un joven cuyo talante quijotesco es el resultado de una educación fallida, dispuesto a vincular la realidad a sus lecturas sin transformar la primera radicalmente; a imprimir un sesgo literario al mundo en el que el protagonista se inscribe. ${ }^{\text {II }}$

II Pese a este matiz, no debemos olvidar que las concomitancias entre Waverley y Don Quijote siguen siendo poderosas, ya que los dos aprehenden la realidad bajo el filtro de sus lecturas, y ambos miran nostálgica y literariamente a un pasado ya fuera del tiempo de la acción que transcurre en las novelas de Cervantes y Scott. Además, cabría destacar que el retrato de la educación de Edward Waverley se enmarca dentro de toda una tradición dieciochesca de Quijotes jóvenes cuya educación fallida es retratada al detalle. Arabella, en el Female Quixote (1752) de Charlotte Lennox, o Don Sylvio von Rosalva, en Die Abenteuer des Don Sylvio von Rosalva (1764), de Christoph Martin Wieland, son claros ejemplos de esta tradición, que Scott conocía bien, tal y como Moro ha demostrado en otro lugar (Transformaciones 264- 
La quijotesca tendencia de Waverley a interpretar románticamente la realidad que le rodea adquirirá una nueva dimensión una vez que se inclina por la carrera militar y es enviado a uno de los regimientos de dragoons acuartelados en Escocia. Como ha subrayado Martin Cusac, el viaje a un contexto cultural desconocido para el protagonista es un motivo que Scott emplea a lo largo de su producción novelística, y que le permite introducir un contraste entre culturas esencialmente distintas:

There are two methods which Scott employs to introduce the contrast of cultures into his novels. One method, perhaps the more striking, requires that the protagonist undertake a journey from his native cultural milieu into one essentially foreign to him. [...] The other method depicts the protagonist in relation to contrasting cultures which exist more or less contiguously. [...] In both groups of narratives the protagonist serves as the means by which the author can present the contrast. (84; énfasis añadido)

El viaje a Escocia, por lo tanto, se erige como una peregrinación hacia lo desconocido. Al abandonar Inglaterra, Edward Waverley pasa a convertirse en espectador de una realidad y una cultura que le son ajenas, y que va a mirar con ojos románticos, bajo el filtro de sus lecturas y de la educación recibida en Waverley Hall. Como ha destacado Pardo, Scott convierte a Edward Waverley en un tipo de turista muy especial: en un turista quijotesco, que observa su destino según sus lecturas de carácter romántico (I33). ${ }^{\mathrm{I2}}$ Sin embargo, su periplo escocés no va a ser única-

319). Friedhlelm Marx ha analizado con gran acierto la figura del joven lector con aspiraciones heroicas y sus vínculos con Don Quijote en su ensayo Erlesene Helden: Don Sylvio, Werther, Wilhelm Meister und die Literatur.

I2 "Waverley ejemplifica el mismo proceso de mediación literaria o pictórica en la percepción de un paisaje: viaja por un territorio no solo físico sino mental, no solo histórico sino imaginativo, recorre un espacio discursivizado e internalizado. Combe y Scott representan las dos versiones más acabadas, cómica y seria, literal y desplazada, anti-romántica y romántica, de esta figura que emerge en los albores del siglo XIX a la que hemos denominado el 
mente un viaje geográfico hacia lo desconocido y lo romántico, sino que, tal y como ha señalado Richard Humphrey, el protagonista de la novela de Scott también va a entrar en contacto con una realidad históricamente menos desarrollada: su viaje se va a constituir como un viaje en el tiempo: "And above all, however, Edward's journey could not have been what it also and decisively is-not just a geographical journey to a far-flung region, but a temporal journey back to an earlier stage of society." (IO-II). De esta manera, Scott emplea al protagonista de su novela como un vehículo para mostrar el contraste entre una sociedad avanzada como la inglesa, de la que Waverley proviene, y un territorio, el escocés, caracterizado por unas estructuras feudales y patriarcales que generan una sensación de otredad tanto en el protagonista como en el lector. El característico héroe mediocre de las novelas de Scott, gracias a su mirada fascinada por lo romántico y por un pasado caballeresco en vías de extinción, se constituye como un mediador entre el presente y el pasado, introduciendo "historical and topographical detail" (Welsh, Hero 34-35). Las peculiares circunstancias educativas que Scott se encarga de reproducir al detalle en los cinco primeros capítulos de su novela y su efecto sobre el quijotesco protagonista de Waverley propician que Edward adquiera el carácter de intermediario "between Lowland and Highland, change and stasis, tomorrow and yesterday" (Humphrey 57; énfasis añadido). La nostálgica mirada romántica de Waverley va a permitir a los lectores de la novela apreciar el contraste entre la belleza caballeresca de un mundo que se resiste a morir, epitomizado por la Escocia jacobita y tribal que fascinará al protagonista, y su necesario carácter decadente en una sociedad fundamentalmente burguesa en la que la realidad que los jacobitas pretenden resucitar ya no tiene cabida. Edward Waverley se convierte, de este modo, en una suerte de "neutral

viajero quijotesco. Este es un personaje que no se limita a desplazarse de un lugar a otro en seguimiento de las empresas que guían su acción [...] sino que es turista en el sentido de que viaja para conocer un territorio respecto del cual asume una distancia estética; y es quijotesco porque lo hace pertrechado de nociones adquiridas por los libros [...]. El viaje es concebido literaria o artísticamente, el quijotismo como una forma literaria o discursiva de viajar" (Pardo I33). 
ground, upon which the opposing elements of the conflict can meet" (Cusac 8I-82), en el instrumento perfecto para reflejar el proceso de declassing del que Lukács hablaba en su tratado sobre la novela histórica. El quijotismo que Scott se afana en explicitar en estos primeros capítulos de su novela se constituye como una herramienta estructural de carácter primordial a la hora de retratar este proceso, en el que la confrontación dialógica entre la mirada de Waverley y la visión más sobria del narrador va a ser fundamental a la hora de apreciar este contraste entre un tiempo caduco y unas nuevas circunstancias históricas (Hartveit 8O-8I; Kerr 29-3I). La naturaleza quijotesca del protagonista, inclinada hacia lo pretérito, es por lo tanto esencial en la construcción de esta antítesis capital en el género histórico.

El retrato del efecto romantizador de la mirada de Waverley sobre la realidad escocesa no se hace esperar. En el capítulo octavo del primer volumen, el narrador relata las primeras impresiones de Waverley en su viaje por Escocia. Al llegar a los dominios del barón de Bradwardine, hacia donde se dirige por recomendación de su tío, amigo de este viejo jacobita escocés, Waverley, pese a la miseria de la aldea cercana a la mansión de Tully-Veolan, no deja de apreciar como las tres o cuatro aldeanas locales se asemejan a "Italian forms of landscape", ignorando la extrema pobreza de su atuendo y su suciedad, que el narrador se encarga de subrayar (I.8: 32-33). Esta tendencia continúa al llegar a Tully-Veolan, una mansión construida "at a period when castles were no longer necessary, and when Scottish architects had not yet acquired the art of designing a domestic residence" (35; énfasis añadido). El carácter más defensivo que palaciego de la mansión, y la soledad "monástica" (35) del lugar despiertan de nuevo la memoria literaria de Waverley, ya que comienza a fantasear con la idea de que "he had reached the castle of Orgoglio" (I.9: 36), en clara referencia a la bestia que aparece en el canto séptimo de The Fairie Queen (I596) de Edmund Spenser; y al observar a dos domésticas enfrascadas en la colada, el joven inglés trae a su mente las "due donzelette garrule" (I.9: 37) de los jardines de Alcina del Orlando Furioso (I532) de Ludovico Ariosto.

En cualquier caso, la realidad de los Lowlands escoceses a mitad del siglo XVIII pronto ofrecerá a Waverley un renovado combustible para alimentar su imaginación romántica. En el capítulo decimoquinto, la 
paz en la mansión de Bradwardine se ve alterada por una incursión de los highlanders en los territorios del barón, con la consecuente rapiña del ganado del noble escocés. Waverley, en un diálogo bastante cómico con Rose Bradwardine, la hija del barón, no logra comprender el mecanismo de extorsión empleado por Fergus Mac-Ivor, el chieftain local, que, bajo una cantidad determinada de dinero asegura que lo que el propio Waverley llega a definir como una suerte de Jonathan Wild (I.I5: 7I) respete las propiedades del barón. ${ }^{13}$ Una vez que Rose narra a Waverley las causas del conflicto entre el barón y el chieftain, y cómo este en cierta ocasión llegó a saldarse con una escaramuza en la que murieron tres highlanders, con el consecuente funeral acompañado del lamento o coronach de las viudas, el narrador destaca cómo "Waverley could not help starting at a story which bore so much resemblance to one of his own day-dreams" (72; énfasis añadido). La realidad escocesa de mediados del siglo XVIII, con sus constantes disputas entre la pequeña nobleza y los líderes tribales de los Highlands, se antoja muy similar a uno de los sueńos literarios que Waverley "had used to conjure up in his imagination as only occurring in ancient times" (72; énfasis añadido). Edward comprende cómo ya no necesita la literatura para viajar en el tiempo y cómo ni siquiera le es necesario salir de Gran Bretaña para vivir las aventuras a las que su indisciplinada educación le había acostumbrado a sońar y a asociar con tiempos pasados:

It seemed like a dream to Waverley that these deeds of violence should be familiar to men's minds, and currently talked of, as falling within the common order of things, and happening in the immediate neighborhood, without his having crossed the seas, and while he was yet in the otherwise well-ordered island of Great Britain. (I.I4: 73; énfasis añadido)

I3 Jonathan Wild, popularmente conocido como "Thief-Taker General" fue uno de los criminales más célebres de Gran Bretaña durante el siglo XVIII. Henry Fielding inmortalizó su figura en su parodia The Life and Death of Jonathan Wild, the Great (1743), en la que la figura del conocido criminal londinense es empleada por el novelista inglés para criticar a Robert Walpole, primer ministro británico sobre el que recayeron numerosas acusaciones de corrupción durante sus 2I años de gobierno (I72I-I742). 
El contraste entre el orden imperante en la Inglaterra de la que el protagonista proviene, y esta Escocia anclada en el pasado resulta más que evidente y despierta la curiosidad de Waverley por realizar una excursión a la tierra que el joven inglés define como "the land of military and romantic adventures" (I.I4: 72). La embajada de Saunders Sanderson, o Evan Dhu Maccombich en su nombre gaélico, para resarcir al barón a cambio de la restauración de los viejos tributos a Fergus Mac-Ivor, le brindará una oportunidad para abandonarse "to the full romance of his situation", y visitar "the den of some renowned outlaw, a second Robin Hood perhaps, or Adam o'Gordon", ofreciéndole "a fund of circumstances for the exercise of a romantic imagination" (I.I6: 78). El narrador, sin embargo, no deja de tener en cuenta el poco heroico acontecimiento que excita su imaginación: el robo de unas vacas lecheras, que Waverley, de manera no poco significativa, "kept in the back-ground," resaltando el efecto literaturizante de la mirada del joven protagonista (78).

Esta tendencia romantizadora de Waverley se va a acrecentar una vez que se adentra junto a Evan Dhu Maccombich en los Highlands para encontrarse con los bandidos que habían perpetrado el robo de las vacas, y posteriormente en su visita a Fergus Mac-Ivor. Ante el encuentro con el bandido Donald Bean Lean, Waverley había anticipado "a stern, gigantic, ferocious figure, such as Salvator [Rosa] would have chosen to be the central object of a group of banditti" (I.I8: 80), pero no encuentra más que una "diminutive and insignificant figure" ataviada en un uniforme francés pasado de moda que incluso tienta la risa de Waverley (8o). Sin embargo, la entrevista con el chieftain no defraudará sus románticas expectativas. El patriarca de la raza de los Ivor impresiona a Waverley por su gracia y dignidad (89), y Mac-Ivor se afanará por acrecentar esta impresión con un banquete a la manera de los Highlands, en el que se evidencia una estructura feudal cerrada en la que todo gira en torno al chieftain, y que Waverley se encarga de trufar nuevamente con referencias a los banquetes de la Odisea de Homero, como el llevado a cabo por los pretendientes de Penélope (I.20: 96-97). La fascinación del protagonista por la patriarcal cultura gaélica de los Highlands será total una vez que entre en escena Flora 
Mac-Ivor, hermana de Fergus, ferviente defensora de la causa Estuardo y traductora de la vieja poesía gaélica al inglés, aspecto que despierta el interés de Waverley. Una vez que ambos se retiran a un pequeńo y estrecho valle para que Flora recite alguna de sus traducciones acompañada de su arpa, el narrador explicita cómo Edward nunca, ni en sus sueños más disparatados, había sido capaz de imaginar una belleza tal, similar a una "fair enchantress of Boiardo or Ariosto" (I.22: IO6). Las lecturas vuelven a definir la percepción de Waverley, que en su romántico viaje hacia una realidad literaria y pretérita se retira a descansar "in that not unpleasing state of mind in which fancy takes the helm, and the soul rather drifts passively along with the rapid and confused tide of reflections that exerts itself to encounter, systematize, or examine them" (II3-I4). Con estas significativas palabras se cierra el primer volumen, en el que este viaje hacia la otredad histórica de Escocia permite a Scott reflejar el quijotesco funcionamiento de la mente romántica de Waverley, que ante el encuentro con una realidad que desconoce cree encontrarse en la tierra de sus lecturas y sueños.

Con el final del primer volumen, en el que Scott retrata con tanta claridad las querencias románticas de Waverley y las peculiares circunstancias históricas que intensifican la actividad imaginativa del protagonista de la novela, el autor escocés va a iniciar un movimiento de sístole y diástole por el que Waverley va a tratar de poner en práctica en un contexto histórico que a priori parece propicio para las gestas románticas las nociones heroicas que su deficitaria educación ha despertado en él; para posteriormente ver estas ideas refutadas por una realidad en la que lo heroico ya no tiene cabida. Este movimiento de sístole y diástole se corresponde de manera bastante exacta con los acontecimientos narrados en el segundo y tercer volumen de la novela, respectivamente. En el segundo volumen, Waverley se va a implicar de lleno en la revuelta jacobita con la esperanza de resucitar el honor de sus antepasados, mientras que en el tercero el narrador va a reflejar con crudeza los desastrosos resultados de esta revuelta, iniciando un proceso de renuncia a lo romántico y de reconocimiento por parte de Waverley de la profunda inadecuación histórica de la causa jacobita. Los encuentros de Waverley en el segundo volumen con los principa- 
les actores de la revuelta, Fergus Mac-Ivor y el pretendiente Estuardo, Charles Edward Stuart (I720-I788); así como con el coronel Talbot, representante del bando gubernamental, en el tercero, apuntalan este movimiento. Fergus y el Chevalier, dos personajes con una clara aureola romántica, símbolos de un orden social ya extinto, excitarán la imaginación de Waverley; el coronel Talbot, heraldo de los tiempos modernos, ayudará a Waverley a reconocer su verdadero ser y reconciliarse con su momento histórico. ${ }^{\mathrm{I}} \mathrm{Al}$ igual que en Don Quijote, en Waverley nos encontramos con una dicotomía clara entre personajes fuera y dentro de la historia, y los encuentros del protagonista con ambos van a definir un movimiento de transición entre un quijotismo fruto de la inexperiencia y una madurez que sólo puede ser obtenida a través de la dolorosa confrontación con los desastres de la guerra. ${ }^{\text {Is }}$

Scott introduce a los lectores en la trama política de su novela a lo largo del segundo volumen. En este, el autor escocés refleja cómo los

I4 Lars Hartveit, en su magnífico estudio A Dream within a Dream: $A$ Thematic Approach to Scott's Vision of Fictional Reality (1974) ha señalado cómo el encuentro con los distintos personajes de la novela juega un papel fundamental en el desarrollo personal de Waverley. Para el estudioso noruego, los personajes que Waverley encuentra en su periplo escocés pueden ser agrupados en dos categorías fundamentales. Por una parte estarían aquellos personajes que renuevan y fomentan sus anhelos románticos y heroicos, por otra los personajes que tratan de cercenar estos impulsos, ofreciendo al protagonista un retrato más exacto de la realidad. En este estudio, por limitaciones de espacio, me centraré únicamente en Fergus Mac-Ivor, Carlos Eduardo Estuardo y en el coronel Talbot como ejemplos arquetípicos de estas dos tendencias, que como bien señala Hartveit pueden ser rastreadas a lo largo de toda la novela.

is El movimiento de transición entre la inexperiencia y la experiencia que tiene lugar en Waverley invita a considerar la novela de Scott como un Bildungsroman o novela de formación. La temática educacional de la novela de Scott ha sido generalmente reconocida por la crítica. Ya en I95I Stewart Gordon señalaba el claro énfasis en la educación de la novela de Scott, señalando cómo el diseño "unificado" de la novela se basa en la fallida educación del protagonista (I07), mientras que estudiosos posteriores como Hart (I966), Sroka (1980), Wolpers (1986), o Moro también han incidido en las afinidades entre Waverley y la tradición del Bildungsroman (Transformaciones 264-319). 
principales actores de la revuelta jacobita en la novela, Fergus Mac-Ivor y Carlos Eduardo Estuardo, conocen el talante quijotesco de Waverley y lo utilizan de una manera muy astuta para atraer al joven noble inglés hacia su causa. ${ }^{16}$ Este proceso comienza una vez que Waverley recibe la notificación de su degradación por ausencia injustificada-y obviamente por su asociación con jacobitas célebres como Fergus Mac-Ivor-en el regimiento de dragoons en el que se encontraba sirviendo. El chieftain aprovecha el enfado de Waverley ante la notificación para sugerirle "an honourable road to speedy revenge" (2.2: 128) y, una vez que el joven inglés comienza a considerar la opción de sumarse a la revuelta jacobita -no sin antes ser advertido por Flora Mac-Ivor de las consecuencias que su implicación tendría y de las razones del interés de su hermano para que se sume al levantamiento-, Fergus le invita a acompañarle a la corte, donde podrá apreciar "a sight worth all the tirades of your romances" (2.3: I3I). En cualquier caso, las palabras de Flora Mac-Ivor, que cuenta con un celo puramente idealista por la causa, propician una reflexión de Waverley sobre la adecuación histórica de la empresa de los Estuardo, y atemperan su entusiasmo inicial por su causa:

Whatever were the original rights of the Stuarts, calm reflection told him, that, omitting the question how far James the Second could forfeit those of his posterity, he had, according to the united voice of the whole nation, justly forfeited his own. Since that pe-

i6 Este proceso es evidente ya en el primer capítulo del segundo volumen. Tras ser herido en una cacería y tener que ser llevado en volandas en una camilla por numerosos highlanders, Fergus cita exactamente las palabras pronunciadas por Don Quijote a la vuelta de su accidentada primera salida (I.5: 57), subrayando cómo adapta su lenguaje a los oídos románticos de Waverley: "Fergus began immediately, with his usual high spirits, to exclaim, "Open your gates, incomparable princess, to the wounded Moor Abindarez, whom Rodrigo de Narvaez, constable of Antiquera [sic], conveys to your castle; or open them, if you like it better, to the renowned Marquis of Mantua, the sad attendant of his half-slain friend, Baldovinos of the mountain. - Ah, long rest thy soul, Cervantes! without quoting thy remnants, how should I frame my language to befit romantic ears!" (2.I: I2I). 
riod, four monarchs had reigned in peace and glory over Britain, and exalting the character of the nation abroad, and its liberties at home. Reason asked, was it worthwhile to disturb a government so long settled and established, and plunge a kingdom into all the miseries of civil war, to replace upon the throne the descendants of a monarch by whom it had been willfully forfeited? (2.5: I4O-4I)

El reconocimiento del carácter anacrónico de las reclamaciones dinásticas de los Estuardo lleva a Waverley a la resolución de volver a su regimiento, pero una reyerta en la aldea de Cairnvreckan, en la que es detenido como sospechoso de sedición y su adolescente empecinamiento en no seguir el consejo de las autoridades locales, que le sugieren descubrir a Fergus Mac-Ivor y reconocer su asociación con este cómo un error propiciado por la juventud "misled by the wild visions of chivalry and imaginary loyalty" (2.9: I63; énfasis añadido), cambiarán radicalmente el rumbo de los acontecimientos. Este momento supone un auténtico punto de inflexión en este segundo volumen, ya que, a partir de su detención en Cairnvreckan, la adopción de la causa jacobita por parte de Waverley va a ser clara, siendo muy significativamente asociada a unas nociones heroicas derivadas de la conciencia del pasado glorioso de los Waverley:

A sentiment of bitterness rose in his mind against the government, which he considered as the cause of his embarrassment and peril, and he cursed internally his scrupulous rejection of Mac-Ivor's invitation to accompany him to the field. "Why did not I," he said to himself, "Like other men of honour, take the earliest opportunity to welcome to Britain the descendant of her ancient kings, and lineal heir of her throne? [...] I had there been free and in arms, fighting, like my forefathers, for love, for loyalty and for fame. (2.10: ı66; énfasis añadido)

La vinculación entre la educación quijotesca recibida por el protagonista en Waverley Honour y la causa jacobita resulta, por lo tanto, bastante clara. La noción de un pasado familiar heroico y la cosmovi- 
sión romántica de Waverley van a ligar al protagonista de la primera novela de Scott a un movimiento político cuyo carácter anacrónico había sido puesto de relieve por él mismo justo antes de su detención en Cairnvreckan. Este entusiasmo por la causa del chevalier, apelativo por el que Carlos Eduardo Estuardo era conocido entre sus seguidores, va a ser total una vez que Waverley es rescatado por un grupo de highlanders de Fergus Mac-Ivor y llevado al palacio de Holyrood en Edimburgo, recuperado por el pretendiente jacobita. Tras su rescate, Mac-Ivor presenta a Waverley ante Carlos Eduardo, que muy astutamente recomienda al joven inglés seguir los pasos de su antepasado Sir Nigel y abrazar "a cause which has little to recommend it but its justice, and follow a prince who throws himself upon the affections of his people to recover the throne of his ancestors, or perish in the attempt" (2.17: 193). La invitación a participar en esta "gallant enterprize" (I93), y la propia figura del chevalier fascinan a Waverley, quien dejándose llevar por su tendencia quijotesca y por los prejuicios de su educación, acepta comprometerse en una empresa caballerescamente atractiva, pero claramente fuera de su momento histórico:

Unaccustomed to the address and manners of a polished court, in which Charles was eminently skillful, his words and kindness penetrated the heart of our hero, and easily outweighed all prudential motives. To be thus personally solicited by a prince, whose forms and manners as well as the spirit which he displayed in this singular enterprize, answered his ideas of a hero of romance; to be courted by him in the ancient halls of his paternal palace, recovered by the sword which he was already bending towards other conquests, gave Edward, in his own eyes, the dignity and importance which he had ceased to consider his attributes. Rejected, slandered, and threatened upon the one side, he was irresistibly attracted to the cause which the prejudices of education, and the political principles of his family, had already recommended as the most just. These thoughts rushed through his mind like a torrent, sweeping before them every consideration of an opposite tendency [...] and Waverley, kneeling 
to Charles Edward, devoted his heart and sword to the vindication of his rights! (2.17: 193; énfasis añadido)

El posterior baile, en el que las doncellas admiran a un "gallant and handsome young Prince, who threw himself upon the mercy of his countrymen, rather like a hero of romance than a calculating politician" (2.20: 206; énfasis añadido), y el rechazo de Flora a casarse con Waverley al considerar que este nunca podrá luchar de manera ideal y sincera por su causa (208), renuevan la determinación del protagonista de Scott de distinguirse en el campo de batalla al servicio de un príncipe que se asemeja a uno de los personajes de aquellas crónicas de Froissart o de la Noue tan presentes en la biblioteca gótica de Waverley Hall. En este segundo volumen, por lo tanto, la fascinación por Fergus Mac-Ivor y por Carlos Eduardo Estuardo vincula al protagonista de Scott con un movimiento político que pretende parar el reloj y devolver a Gran Bretaña a la situación previa a la Revolución Gloriosa. El encuentro de Waverley con Fergus Mac-Ivor y con el príncipe Estuardo sitúa al protagonista fuera de la historia, y es este el momento de la novela en el que su quijotismo resulta más exaltado y evidente.

En cualquier caso, el segundo volumen de la novela no se cierra con la exaltación romántica de la corte de Carlos Eduardo Estuardo. A lo largo de los últimos tres capítulos del volumen, Scott se preocupa de mostrarnos la marcha jacobita hacia el sur, y en el retrato que el autor escocés hace de ella nos vamos a encontrar con una visión más sobria sobre la verdadera situación de los rebeldes: la visión del narrador, que inicia el movimiento de diástole hacia la renuncia de lo romántico por parte de Waverley en estas últimas páginas del segundo volumen. Este proceso es evidente en el vigesimoprimer capítulo. Desde las alturas de St. Leonard's Hill, el narrador introduce a Waverley observando al ejército jacobita iniciar la marcha. Lo que en un principio parece una escena de claro corte romántico, en un paisaje dramático y con los montañeros de los Highlands organizados en clanes, con sus respectivos pendones y marcha de gaitas, desfilando liderados por el chevalier y el estandarte con su emblema Tandem Triumphans (2.21: 213), se ve pronto matizado por una mirada más sobria y más detallada, capaz de 
vislumbrar no sólo las luces, sino también las sombras de las huestes jacobitas. Junto a los familiares directos de los chieftains, todos ellos profusamente armados, el narrador destaca a una segunda fila de campesinos, mal equipados y mostrando una extrema penuria, no en vano el narrador los llega a asemejar a "bandidos" (2.21: 215). Esta imagen causa el asombro de Waverley, que hasta ese momento sólo conocía la magnificencia tribal de las tropas que Fergus Mac-Ivor le había mostrado de manera muy selectiva, y el narrador destaca cómo no resulta ninguna sorpresa que el protagonista de la novela "should have felt damped and astonished at the daring attempt of a body not then exceeding four thousand men, and of those not above half the number, at the utmost, well armed, to change the fate, and alter the dynasty, of the British kingdoms" (2.2I: 2I5). Por primera vez en toda la novela, la rebelión jacobita es desenmascarada como una empresa desesperada y profundamente quijotesca.

Este inicio del proceso de agnición de Waverley en este último sector del segundo volumen va a verse acentuado una vez que uno de los acompańantes ingleses de Waverley en su regimiento de dragoons muere en una escaramuza previa a la batalla de Prestonpans, en la que los jacobitas obtienen una importante victoria (2.22: 218-19). En esta, al reconocer a su antiguo regimiento en el ejército enemigo, Waverley comprende por fin cómo su lealtad a Carlos Estuardo no es más que el producto de un sueño caballeresco que poco tiene que ver con su identidad real y con el momento histórico al que pertenece:

It was at that instant, that looking around him, he saw the wild dress and appearance of his Highland associates, heard their whispers in an uncouth and unknown language, looked upon his own dress, so unlike that which he had worn from his infancy, and wished to awake from what seemed at the moment a dream, strange, horrible, and unnatural. "Good God," he thought, "am I then a traitor to my country, a renegade to my standard, and a foe, as that poor dying wretch expressed himself, to my native England!" (2.23: 22I) 
Con este despertar del sueño quijotesco de Waverley y con la batalla de Prestonpans, en la que Waverley logra salvar a un oficial inglés, pero presencia la muerte cruel y violenta del general de su regimiento de $d r a-$ goons en el fragor de la batalla (2.24: 226), se cierra el segundo volumen de la novela, iniciando el movimiento de diástole que va a reconciliar a Waverley con Inglaterra y con su realidad histórica. El encuentro con el coronel Talbot, precisamente el oficial al que Waverley logra salvar, y que se va a erigir como un claro representante de los caballeros de los nuevos tiempos, va a ser fundamental en este proceso.

El coronel inglés Talbot es introducido por Scott al inicio del tercer volumen, concretamente en el segundo capítulo. En Talbot, el protagonista encuentra a un tipo de militar que difiere radicalmente de todo lo encontrado en Escocia. A través de la introducción del coronel inglés, Scott nos muestra el retrato viviente de un hombre de armas de un tiempo nuevo, un momento histórico distinto:

As a specimen of the military character, Talbot differed from all whom Waverley had as yet seen. The soldiership of the Baron of Bradwardine was marked by pedantry; that of Major Melville by a sort of martinet attention to the minutiae and technicalities of discipline, rather suitable to one who was to manoeuvre a battalion, than to him who was to command an army; the military spirit of Fergus was so much warped and blended with his plans and political views, that it was that of a petty sovereign, rather than of a soldier. But Colonel Talbot was in every point the English soldier. His whole soul was devoted to the service of his king and country, without feeling any pride in knowing the theory of his art with the Baron, or its practical minutiae with the major, or in applying his science to his own particular plans like the Chieftain of Glennaquoich. Added to this, he was a man of extended knowledge and cultivated taste, although strongly tinged, as we have already observed, with those prejudices which are peculiarly English. (3.5: 246) 
Las entrevistas de Waverley con el coronel van a suponer un auténtico correctivo epistemológico para el protagonista de la novela, que gracias al militar inglés comenzará a experimentar las consecuencias de su implicación en la revuelta jacobita. Talbot, que advierte a Waverley sobre cómo las tropas jacobitas han iniciado una empresa "to which your strenghth appears wholly inadecuate" (3.2: 233), comunica a Edward las represalias que su familia ha experimentado por su implicación en la revuelta, como la degradación inmediata de Richard Waverley de cualquier responsabilidad gubernamental y el apresamiento de su tío por las sospechas de sedición despertadas por la asociación de Waverley con los rebeldes y por su antiguo historial de apoyo a los jacobitas (235). Este carácter de correctivo epistemológico continúa en el capítulo cuarto, en el que Talbot clarifica a Waverley todas las maniobras llevadas a cabo por Donald Bean Lean para implicarle en la revuelta, señalando a Waverley cómo se había visto "trepanned into the service of this Italian knight-errant" (3.4: 24I; énfasis añadido). Las posteriores entrevistas entre ambos, en las que Waverley paulatinamente se va mostrando más receptivo a los argumentos del coronel inglés, ejercen un efecto cumulativo una vez que el protagonista de la novela de Scott se ve confrontando no sólo con las secuelas personales que su implicación en el movimiento jacobita acarrea a su familia, sino también con los efectos de la derrota de Carlisle, en la que los jacobitas son barridos por un ejército bien equipado, moderno y con un claro sentido de la táctica militar; y, sobre todo, a causa de las consecuencias colectivas que la quijotesca empresa de Carlos Estuardo ocasiona: los desastres de la guerra.

Waverley comienza a apreciar los efectos de la contienda al escapar muy afortunadamente de una escaramuza en la que Fergus Mac-Ivor es apresado por las tropas inglesas (3.12: 276). Al retornar al lugar de la escaramuza, el protagonista observa "the dead bodies of men and horses, and the usual companions of war, a number of carrion crows, hawks and ravens" (3.I4: 28I). Las aves carroñeras y la presencia de los numerosos cadáveres comienzan a despertar al joven entusiasta inglés de sus sueños románticos de heroísmo. Al permanecer en Ullswater, donde tiene que detenerse por una fuerte nevada que impide su marcha du- 
rante algunos días, Waverley empieza a aceptar de manera definitiva los argumentos del coronel Talbot, culminando el proceso de corrección epistemológica que se inició en los últimos compases del segundo volumen y que alcanza en este momento su punto culminante:

In this solitary and secluded situation, without the advantage of company or conversation with men of cultivated minds, the arguments of Colonel Talbot often recurred to the mind of our hero. A still more anxious recollection haunted his slumbers-it was the dying look and gesture of Colonel G-. Most devoutly did he hope, as the rarely-occurring post brought news of skirmishes with various success, that it might never again be his lot to draw his sword in civil conflict. [...] These reveries he was permitted to enjoy, undisturbed by queries or interruption, and it was in many a winter walk by the shores of Ullswater, that he acquired a more complete mastery of spirit tamed by adversity, and that he felt himself entitled to say firmly, though perhaps with a sigh, that the romance of his life was ended, and that his real history had now commenced. (3.I3: 282-283; énfasis añadido)

Las miserias de la guerra y las consecuencias de la implicación de Waverley en la utopía restaurativa de los jacobitas propician en el joven inglés una renuncia a sus sueños caballerescos y acarrean, en palabras de Lars Hartveit, "the realization that the heroic is a dream, and that any attempt to treat it as a reality is dangerous both on a personal and a national level" (IO7). La experiencia del fracaso por fin permite a Waverley dominar su espíritu, alcanzando la madurez de la que ha carecido claramente a lo largo de toda la novela. Este es, por lo tanto, el punto culminante de la narrativa, aquel al que esta se ha dirigido desde los primeros capítulos en los que se narraba la quijotesca educación de Waverley. Una de las últimas entrevistas con Talbot confirma este proceso, no en vano, el protagonista afirma al coronel cómo se encuentra "heartily tired of the trade of war", ya que, pese a la fascinación que en sus lecturas había sentido por "the plumed troops and the big war", la realidad de la contienda, con sus vigilias al raso y sus marchas 
nocturnas "are not at all to my taste in practice" (3.15: 290). Como destaca Levine, esta última entrevista con Talbot denota cómo "the great chivalric ideals of heroism are turned to dream" (I49), transformando a Waverley, como significativamente subraya el narrador sirviéndose de una cita de The Rhyme of the Ancient Mariner de Samuel Taylor Coleridge, en "a sadder, and a wiser man [...], a compensation for the gay dreams which in his case experience had so rapidly dissolved" (3.I6: 296). Cabría añadir que este proceso de agnición no solo permite a Waverley recuperar su identidad y conocerse a sí mismo, sino que sobre todo le convierte, como a don Quijote tras su renuncia a su empresa caballeresca, en un hombre reconciliado con su momento histórico, en un caballero ya situado dentro de la historia.

ConClusión: WAVERLEY, LA TRADICIÓN CERVANTINA Y LA NOVELA HISTÓRICA

De lo anteriormente expuesto se pueden extraer una serie de conclusiones relacionadas, por una parte, con la clara herencia cervantina de Waverley, or 'tis Sixty Years Since, y, por otra, con el papel que juega Don Quijote en la configuración estructural de esta novela, habitualmente considerada la primera novela histórica de la literatura europea.

En lo que se refiere a la filiación cervantina de Waverley, cabe destacar que Scott, tal y como manifiesta en el tercer capítulo de la novela, sigue a Cervantes, pero no trata de imitar al pie de la letra el modelo que Don Quijote le ofrece. El novelista escocés no presenta a un viejo enjuto y demente capaz de transformar dentro de su mente la realidad que le rodea, sino que expone al lector un proceso mucho más complejo al reflejar las consecuencias de una educación romántica y la consecuente atracción por un mundo históricamente anacrónico que se asemeja a las lecturas que marcan la educación del protagonista. Tal y como el propio autor escocés destacaba en su comentario sobre el Launcelot Greaves de Smollett, el fenómeno del quijotismo ha de inscribirse en un lugar y en unas coordenadas históricas que permitan albergar el sueño de restaurar un tiempo pretérito. En otras palabras, 
para Scott, el quijotismo está directamente vinculado a un horizonte referencial que posibilita la asociación entre las lecturas y la realidad, y no todos los momentos históricos pueden albergar esta conjunción. En este sentido, la elección de la Escocia de las revueltas jacobitas a mediados del siglo XVIII no resulta aleatoria. Los intentos jacobitas por restituir una dinastía y un orden social fuera de su momento histórico ofrecen ese vínculo entre las lecturas y la realidad, ese horizonte referencial que Scott quería ver en la España de finales del siglo XVII en la que Don Quijote se inscribe. El fenómeno del quijotismo se ve de esta manera doblemente plausibilizado, ya que, por una parte, Scott ofrece un retrato muy detallado de las causas de ese quijotismo, algo por lo que Cervantes pasa muy por encima en su obra; y, por otra, nos muestra un momento de transición histórica en el que el establecimiento de un vínculo entre unas lecturas caballerescas y la realidad todavía resulta posible. El autor de Waverley se afana en retratar cómo la Escocia de "Sixty Years Since", aquella que le ofrece la posibilidad de preservar "some idea of the ancient manners of which I have witnessed the almost total extinction" (3.25: 340), supone un horizonte referencial ideal para el sueño caballeresco de Edward Waverley, muy al contrario que la Inglaterra dieciochesca del Launcelot Greaves de Smollett, introduciendo de esta manera un matiz inédito en la tradición cervantina al retratar el fenómeno del quijotismo como el resultado parcial de un momento histórico concreto, variación que inaugura una senda que novelistas posteriores explorarán a lo largo del siglo XIX. Waverley se constituye, por lo tanto, no como una mera imitación de Don Quijote, sino como una de las más innovadoras transformaciones que la novela cervantina experimenta durante su dinámica recepción en las letras británicas de los siglos XVIII y XIX.

Por otra parte, el influjo de Don Quijote en Waverley conlleva una serie de consecuencias directas en lo que se refiere al alumbramiento del género histórico, del que la novela de Scott es habitualmente considerada el primer espécimen. Como reflejan los comentarios de Scott sobre Don Quijote en sus Essays on Chivalry, Romance, and the Drama, el novelista escocés comprendió la novela cervantina como el reflejo de un periodo de transición entre un mundo caballeresco que se resistía a morir y la realidad de Espańa a comienzos del siglo XVII, en la que la 
empresa quijotesca debía de resultar necesariamente anacrónica. Como se ha tratado de reflejar a lo largo de estas páginas, el fenómeno del quijotismo en Waverley se erige como la principal fuente de atracción hacia ese pasado que se resiste a morir, como una mirada que trata de encontrar en la realidad un universo en vías de extinción. El carácter quijotesco de Waverley va a propiciar un movimiento de ida y vuelta en el que el protagonista de la novela cree encontrar sus sueños caballerescos en la realidad, para posteriormente ver estos sueños desarmados por la pesadilla del conflicto bélico. El quijotismo juega un papel fundamental en este proceso, y la fascinación del joven inglés por los tiempos ya pasados va a permitir que el protagonista de la novela se convierta en el medio que Scott utiliza para articular un diálogo entre lo antiguo y lo nuevo, entre los románticos caballeros de la Escocia jacobita y los heraldos de una época no tan romántica que relega lo heroico al ámbito de lo estético, de lo literario. El contraste entre los caballeros de tiempos pasados y los caballeros de tiempos modernos, tan fundamental en Don Quijote y en el proceso de agnición que el hidalgo manchego experimenta en la novela de Cervantes, adquiere, como he tratado de demostrar en estas páginas, una importancia similar en la novela de Scott, y epitomiza la confrontación entre movimientos históricos y sociales antagónicos que Lukács consideraba el principio estructural básico de la novela histórica. Quizás, la sombra de Cervantes sea aún más alargada de lo que hemos asumido, y probablemente haya llegado el momento de subrayar el decisivo papel que Cervantes y su Quijote juegan en el alumbramiento de uno de los géneros novelescos más populares a lo largo de los dos últimos siglos.

\section{Universidad DE CANTABRia alfredo.moro@unican.es}

\section{Obras citadas}

Álvarez, Román. Origenes y evolución de la novela histórica inglesa. Salamanca: Ediciones Universidad de Salamanca, 1983.

Byron, Lord. The Major Works. Ed. Jerome K. McGann. Oxford: Oxford UP, 2008. Cátedra, Pedro. El sueño caballeresco: De la caballería de papel al sueño real de Don Quijote. Madrid: Abada Editores, 2007. 
Cervantes, Miguel. Don Quijote de la Mancha. Ed. Francisco Rico. Madrid: Alfaguara, 2004.

Cusac, Martin. Narrative Structure in the Novels of Sir Walter Scott. La Haya: Mounton, 1969.

De Groot, Jerome. The Historical Novel. Albingdon: Routledge, 2010.

Endress, Heinz-Peter. Los ideales de Don Quijote en el cambio de valores de la Edad Media al Barroco. Pamplona: EUNSA, 2000.

Gerli, Michael. “Pray landlord, bring me those books': Notes on Cervantes, Walter Scott and the Ethical Legitimacy of the Novel in Early Nineteenth Century England." Corónente tus hazañas: Studies in Honor of John Jay Allen. Ed. Michael J. McGrath. Newark: Juan de la Cuesta, 2005. 23I-43.

Hamnett, Brian. The Historical Novel in Nineteenth-century Europe: Representations of Reality in History and Fiction. Oxford: Oxford UP, 2015.

Hart, Francis R. Scott's Novels: The Plotting of Historic Survival. Charlottesville: UP of Virginia, 1966.

Hartveit, Lars. A Dream within a Dream: A Thematic Approach to Scott's Vision of Fictional Reality. Oslo: Universitetsforlaget, 1974.

Hegel, Georg Wilhelm Friedrich. Werke. Fráncfort: Suhrkamp Verlag, 1986.

Humphrey, Richard. Walter Scott: Waverley. Cambridge: Cambridge UP, 1993.

Keen, Maurice. La caballería. Barcelona: Ariel, 1986.

Kerr, James. Fiction against History: Scott as Storyteller. Cambridge: Cambridge UP, 2007.

Levine, George. "Sir Walter Scott: The End of Romance." Wordsworth Circle Io (1979): I47-I60.

Lockhart, John Gibson. "Life of Cervantes." The History of the Ingenious Gentleman Don Quixote of la Mancha. Translated from the Spanish by Motteux. A New Edition with Copious Notes and an Essay on the Life and Writings of Cervantes. In Five Volumes. Edimburgo: Archibald Constable, I822.

Lukács, Georg. The Historical Novel. Lincoln: U of Nebraska P, 1983.

Martínez Bonati, Félix. EL QuiJOTE y la poética de la novela. Alcalá de Henares: Centro de Estudios Cervantinos, I995.

Mancing, Howard. "The Quixotic Novel in British Fiction of the Nineteenth and Twentieth Centuries." The Cervantean Heritage: Reception and Influence of Cervantes in Britain. Ed. J. A. G. Ardila. Londres: Legenda, 2009. I04-I7.

Marx, Friedhelm. Erlesene Helden: Don Sylvio, Werther, Wilhelm Meister und die Literatur. Heidelberg: Carl Winter, 1995.

McDonald Jr, W.U. "Scott's conception of Don Quixote." Midwest Review (1959): 37-42.

Menéndez Pidal, Ramón. Cervantes y el ideal caballeresco. Discurso leído por el Excmo. Señor Ramón Menéndez Pidal, Director de la Real Academia de la Lengua Española en la sesión de clausura de la Asamblea Cervantina de la Lengua Española. Madrid: Patronato del IV Centenario del Nacimiento de Cervantes, I948. 
Moro Martín, Alfredo. Transformaciones del Quijote en la novela inglesa y alemana del siglo XVIII. Alcalá de Henares: Universidad de Alcalá, Servicio de Publicaciones, 2016.

-. "Cervantismos olvidados: Sir Walter Scott" en Comentarios a Cervantes. Actas selectas del VIII Congreso Internacional de la Asociación de Cervantistas. Oviedo: Fundación María Cristina Masaveu Peterson, 20I5.

Müllenbrook, Heinz Joseph. "Scotts Waverley als 'Respons' aufCervantes's Don Quixote." Literaturwissenschaftliches Jahrbuch im Auftrage der Görres Gesellschaft, 40 (I999): I39-I54.

Müller, Wolfgang. "Sir Walter Scotts Waverley und die Don Quijote Tradition." Arcadia, 23 (1988): I33-I48.

Pardo, Pedro Javier. "Viajeros quijotescos y viajes cervantinos en las letras británicas" en La cultura española en la Europa Romántica. Ed. José Checa Beltrán. Madrid: Visor Libros, 20I4: I2I-I53.

Riquer, Martín de. "Cervantes y la caballeresca" Summa Cervantina. Ed. Juan Bautista Avalle-Arce y Edward Calverley Riley. Londres: Támesis Book, I973: 273-292.

—. "Introducción" en Don Quijote de la Mancha. Barcelona: Planeta, 1992.

Scott, Walter. Lives of the Novelists. Filadelfia: H.C. Carey, I825.

-. Essays on Chivalry, Romance, and the Drama. Londres: Cadell, I834.

- Waverley, or 'Tis Sixty Years Since. Ed. C. Lamont. Oxford: Oxford UP, 2008.

Snel-Wolfe, Clara. "Evidences of Scott's Indebtedness to Spanish Literature." The Romanic Review 23.4 (1932): 3OI-II.

Sroka, Kenneth. "Education in Walter Scott's Waverley." Studies in Scottish Literature I5 (1980): 139-65.

Stewart Gordon, S. "Waverley and the 'Unified Design." English Literary History I8 (195I): 107-23.

Ter Horst, Robert. "Effective Affinities: Walter Scott and Miguel de Cervantes." Cervantes for the 2Ist Century: Studies in the Honour of Edward Dudley. Ed. F. la Rubia Prado. Newark: Juan de la Cuesta, 2000. 199-220.

Vivar, Francisco. Don Quijote y los caballeros de tiempos modernos. Salamanca: Ediciones Universidad de Salamanca, 2007.

Welsh, Alexander. The Hero of the Waverley Novels. New Haven: Yale UP, 1963.

-. Reflections on the Hero as Quixote. Princeton: Princeton UP, I98I.

Williamson, Edwin. "La transformación de Don Quijote y Sancho en la segunda parte." Cervantes y los cauces de la novela. Ed. Emilio Martínez Mata. Madrid: Visor Libros, 20I4. 33-67.

Wolpers, Theodor. "Der romantische Leser als Kriegheld und Liebhaber: Poetisierung der Realität in Walter Scotts 'Waverley.'" Gelebte Literatur in der Literatur: Studien zur Erscheinungsformen und Geschichte eines literarisches Motivs. Ed. Theodor Wolpers. Gotinga: Vandenhöck, 1986. I85-97. 